\title{
British Undergraduate Dental Research Conference 2021
}

By Sumaya Al-Attar, 4th Year Dental Student and Sabina Rahman, 3rd Year Dental Student, University of Manchester, UK

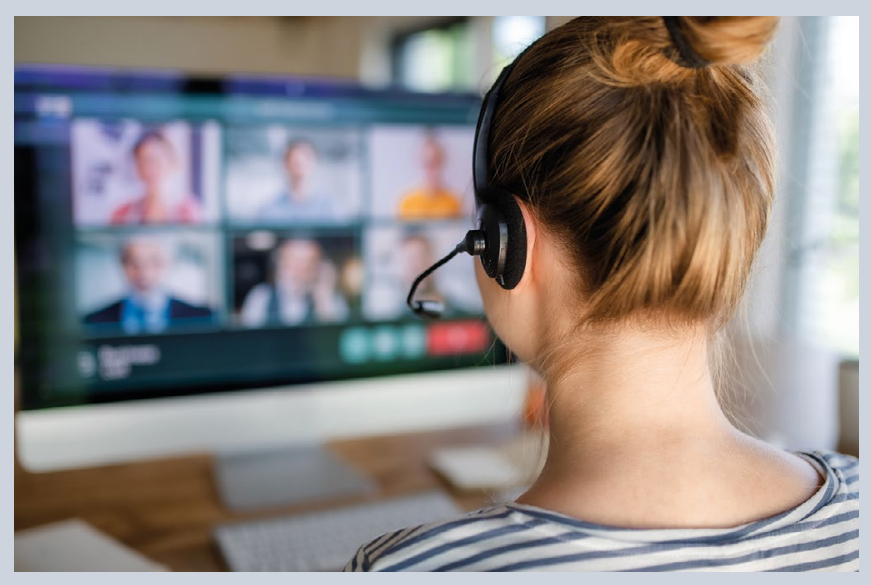

The sixth annual British Undergraduate Dental Research Conference (BUDRC) was held virtually over three consecutive Sundays in March. Tasked with adapting to current COVID restrictions but firm in our aim to promote research, enhance clinical skills and provide a networking platform for future dentists, the Manchester Undergraduate Dental Research Society (MUDRSoc) organised yet another memorable event.

Day 1 commenced with talks by world-renowned speakers such as Professor Jan Clarkson who discussed her Cochrane rapid review on AGPs, followed by student research and clinic case competitions. One hundred percent of delegates enjoyed the event and we received great feedback such as 'Thank you for giving students the opportunity to find out more about research and how it benefits our future careers.

The rest of the conference consisted of innovative virtual hands-on workshops. Day 2 was a suturing workshop where delegates received suturing kits in the post, followed a live tutorial by Dr Kushnerev and practised in small groups with dentists who provided tailored feedback.

Day 3 was a posterior composite workshop where we teamed up with Dr Shiraz Khan and Sculpt Composite to provide home composite kits and a live tutorial of the fast-modelling technique. These sessions were highly popular with feedback such as 'It was really lovely to finally get some hands-on experience as a first year!' and 'The session was so helpful; I'm really excited to implement what I learnt into clinics'.

The conference was a national success, with 300 students from 15 dental schools in attendance. We are pioneering these remote hands-on opportunities, and feedback showed that $96 \%$ of delegates agree that similar sessions should be made part of university curricula. The MUDRSoc committee, including Sumaya Al-Attar, Sabina Rahman, Yasmin Egonu and Mobina Bohloli, created an unforgettable virtual experience and one which may help reshape the future of dental education.

\section{BAOMS President pledges sustainable and fit-for- purpose OMFS training}

British Association of Oral and Maxillofacial Surgeons (BAOMS) 2021 President Austen Smith is using his presidential year to push for a more sustainable, shorter, fit-for-purpose and affordable training pathway for an OMFS career.

Dr Smith, an OMFS Consultant with Sheffield Teaching Hospitals and Barnsley Hospital NHS Foundation Trust, said: 'My aim is to reduce duplication and condense the acquisition of skills into a UK-wide, accessible and consistent route to a career in OMFS.

'Over 23 years of surgical practice in Sheffield and Barnsley I have come to appreciate how critical good training is in a challenging field of surgical care. I want to see straightforward, nationally applied and sustainable training for those who are interested in and commit to this magical specialty.'

Dr Smith recalls: 'I can remember being near-broke in the latter stages of my second degree and will always be grateful to my local authority Kirklees for a student grant that at the time saved me - and also the bank of mum and dad as with many students currently'.
Dr Smith says that historical barriers to improvements in the length of OMFS training have resulted in expensive and overlong duplication of young clinicians' education. He believes that some of the barriers to change will now be removed because EU legislation will no longer affect medical and dental courses.

'BAOMS will encourage training bodies to see the sense and value in new proposals to streamline the overall pathway from new graduate to OMFS consultant.'

He is also committed to protect the interests of Fellows in Training, the BAOMS Associate Membership for junior OMFS trainees: 'Junior colleagues hold key roles in hospital OMFS departments across the UK. They are at an early stage in their careers, and a real change and improvement in the training trajectory could make a real difference to them.

'I don't believe those committing to this critical NHS specialty should have to spend disproportionately in investing in their training. I want to see a leaner, more efficient shape to OMFS training consistently applied across the UK, and that is what I have committed to initiate during my BAOMS Presidency. 